\title{
Isovolumic relaxation sound: a new class of added heart sound?
}

\author{
C H Lee, D G Gibson
}

\begin{abstract}
An early diastolic sound in a patient with apical left ventricular disease is reported. Pulsed Doppler echocardiography showed blood flow within the left ventricular cavity during the isovolumic relaxation period whose peak flow velocity was synchronous with the onset of this diastolic sound. Because it occurred before filling started it could not have been either a third or fourth heart sound. It must thus represent a distinct and apparently unrecognised class of diastolic sound.
\end{abstract}

The third and fourth heart sounds have always been related to blood flow across the atrioventricular valves. When relaxation is asynchronous, however, blood flow can also occur within the ventricle during the isovolumic period. The associated changes in blood flow velocity may be rapid, raising the possibility that enough energy may be dissipated to generate a sound at a time when the atrioventricular valves are closed. We report such a case where flow during isovolumic relaxation period was associated with an added heart sound.

\section{Case report}

A 68 year old woman with aortic stenosis and three vessel coronary artery disease underwent aortic valve replacement and coronary bypass grafting. Postoperatively a left pleural, which had effusion developed and she remained unwell. Cardiomegaly was noted and diastolic added sounds were heard. It was believed that she might have suffered a perioperative infarction, which had impaired left ventricular function.

Two diastolic added sounds were confirmed by phonocardiography, one starting $90 \mathrm{~ms}$ and the other $190 \mathrm{~ms}$ after aortic closure $\left(\mathrm{A}_{2}\right)$ (fig 1A). Cross sectional echocardiography showed apical wall motion abnormality with moderate impairment of basal left ventricular function. Pulsed Doppler, recorded at the chordal level with the transducer at the apex, showed a summation mitral filling pattern: a single flow velocity pulse occurring in early diastole soon after the second heart sound but also after the $\mathbf{P}$ wave on the electrocardiogram. This pulse was preceded by striking flow away from the apex during isovolumic relaxation lasting $150 \mathrm{~ms}$ and reaching a peak velocity of $0.75 \mathrm{~m} / \mathrm{s}$. The timing of its peak velocity was synchronous with the onset of the first diastolic sound (X, fig 1B). That of the
Cardiac Department, Royal Brompton National Heart and Lung Hospital, London CH Lee

D G Gibson

Correspondence to Dr D G Gibson, Cardiac Department, Royal Brompton National Hear and Lung Hospital, Sydney Street, London SW3 6NP.

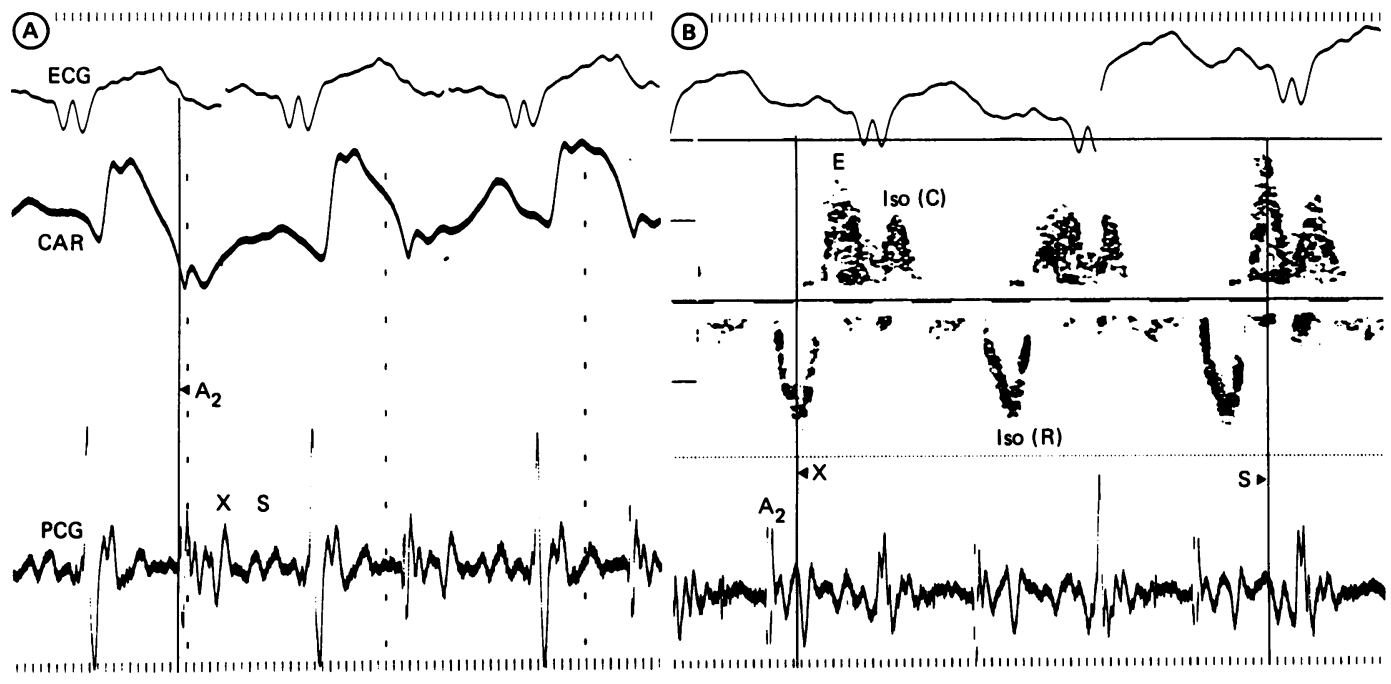

Figure 1 (A) Simultaneous recordings of electrocardiogram (ECG), indirect carotid pulse (CAR), and phonocardiogram (PCG). Aortic closure $\left(A_{2}\right)$ corresponds to the dicrotic notch on the carotid pulse and is followed by two added diastolic sounds ( $X$ and $S$ ). (B) Pulsed Doppler recording at chordal level of the left ventricle for optimal registration of flow velocity during isovolumic relaxation period (Iso $(R)$ ). Early diastolic summation filling velocity registration of flow velocity during isovolumic relaxation period (Iso (R) . Early diastolic summation filling velocity
$(E)$ and flow during isovolumic contraction period (Iso $(C)$ ) are also shown. The onset of the first diastolic added sound $(X)$ after aortic closure $\left(A_{2}\right)$ corresponded in time to the peak isovolumic relaxation flow velocity and that of the second diastolic sound $(S)$ to peak summation wave velocity. Full scale deflection $\pm 2 \mathrm{kHz}$. Time marker 20 ms. 


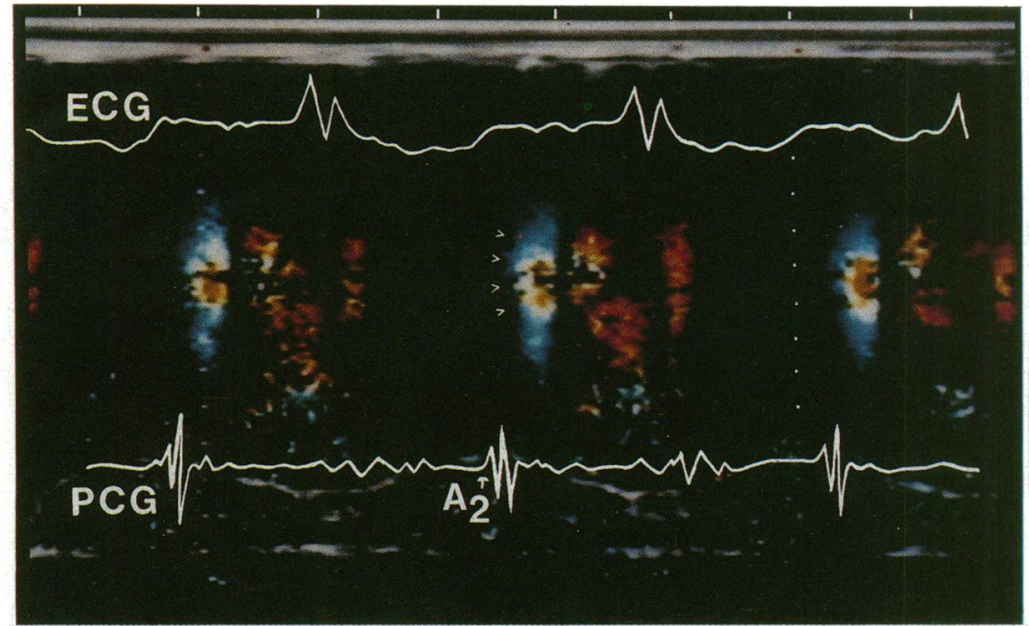

Figure 2 Colour $M$ mode recorded from the apex, directed towards the mitral ring and left atrium, with simultaneous electrocardiogram (ECG) and phonocardiogram $(P C G)$. Forward flow from left atrium to left ventricle is coded in red. Note the abnormal flow, coded blue (arrows), that immediately follows $A_{2}$ and precedes the onset of forward flow. This corresponds to blood flow from apex to base during isovolumic relaxation. For clarity of reproduction, the electrocardiogram and phonocardiogram have been retraced. Depth markers $1 \mathrm{~cm}$, time marker $200 \mathrm{~ms}$.

second diastolic sound (S, fig 1B), $100 \mathrm{~ms}$ later, coincided with peak early diastolic transmitral flow velocity. There was also an abnormal flow signal from base to apex after mitral closure during the isovolumic contraction period, but this was not associated with an additional heart sound (fig 1B). On the right side, peak early diastolic flow velocity across the tricuspid valve was synchronous with that across the mitral valve. Pulmonary valve closure, timed from pulmonary valve artefact, followed $\mathrm{A}_{2}$, by $10 \mathrm{~ms}$ in expiration. The left ventricular flow disturbance was further examined by colour flow mapping. Abnormal flow was centred at the junction between normally moving and diseased segments of the left ventricle and was directed from the apex to the base. Colour $M$ mode confirmed that this flow occurred during the isovolumic relaxation period, lasting for $150 \mathrm{~ms}$ and involving a column of blood $5 \mathrm{~cm}$ in length (fig 2).

\section{Discussion}

A diastolic heart sound is always an important clinical finding in a patient with heart disease. Though there is no consensus on how such sounds are generated, it is generally accepted that they are related in some way to ventricular filling - the third sound to filling in early diastole and the fourth to atrial systole. We, and others, have suggested more specifically that they represent energy dissipated by sudden changes in blood flow velocity. ${ }^{12}$ On this basis, the third sound is synchronous with the transition from an accelerating to a decelerating flow during early diastole, and the fourth sound with the onset of acceleration caused by atrial systole. If this explanation is correct, one might expect diastolic sounds to be generated whenever there is a sudden change in blood flow velocity.

Blood flow within the left ventricle during isovolumic relaxation is a common finding and it has been documented in normal individuals as well as in patients with left ventricular hypertrophy. ${ }^{3}$ Because blood is incompressible, this flow is the natural consequence of a change in left ventricular cavity shape during a period of constant ventricular volume. We have previously analysed such changes in detail as they occur in patients with coronary heart disease. ${ }^{4}$ The change itself is caused by asynchronous relaxation, the onset of outward wall motion being characteristically delayed in segments of the myocardium supplied by diseased coronary arteries. These findings can be directly applied in the present case. The patient had apical disease, where the onsets of tension rise and fall are likely to have been delayed, and thus asynchronous with those at the base of the heart. Such differences in wall tension are the direct cause of the abnormal blood flow we observed, initially from base to apex during isovolumic contraction and reversing during isovolumic relaxation.

The onset of the added heart sound in this patient corresponded with the peak of the flow transient within the ventricle, when there was a sudden change from acceleration towards base to acceleration towards the apex. The added sound thus bore the same time relation to the changing blood flow as the classic third heart sound does to changing blood flow velocities during rapid filling. ${ }^{1}$ It differed from a third heart sound in that it occurred before mitral valve opening, and so could not have been in any way related to rapid ventricular filling. In addition, the patient had a second added sound later in diastole whose onset corresponded with the peak right and left ventricular filling velocities. Because both mitral and tricuspid flow were summation waves this second diastolic sound was a summation rather than a fourth heart sound. Finally, aortic and pulmonary closure were effectively synchronous, which excludes delayed $\mathrm{P}_{2}$ as a possible mechanism. We conclude, therefore, that in this patient the sudden reversal in the direction of blood flow within the ventricular cavity during isovolumic relaxation was the cause of the added sound in our patient.

Asynchronous relaxation and the resulting blood flow within the left ventricular cavity is common in normal hearts as well as in left ventricular hypertrophy and coronary artery disease. A possible associated heart sound, however, has received little attention. In part this may reflect the lamentable disuse into which the valuable art of phonocardiography has fallen. An additional cause is that the timing of the intraventricular blood flow was very abnormal in our patient because isovolumic relaxation itself was prolonged. It is much more common for peak isovolumic velocities to coincide with mitral or aortic valve closure, so that any vibrations they might generate are superimposed on the normal first or second, making a possible additional contribution difficult to document.

Our observation of a loud sound during isovolumic relaxation emphasises that it may be necessary to modify current nomenclature. Rather than calling any low pitched early diastolic sound a "third", we suggest the term 
should be applied only to vibrations occurring during the rapid filling phase of one or other ventricle and "fourth" to those associated with atrial systole. The sound we have described is not covered by either of these terms, and may be better called a "left ventricular isovolumic relaxation sound". Even this more complex term may prove unsatisfactory because the flow velocity associated with it was in the reverse direction to that more commonly seen during isovolumic relaxation. ${ }^{3}$ Nevertheless, its association with a flow reversal within the ventricle provides further evidence that sudden changes in blood velocity are a general mechanism by which diastolic sounds can be produced.

It is thus clear that not all early diastolic sounds, even in patients with obvious left ventricular disease, are third heart sounds. This conclusion may be important when attempts are made to elucidate the pathological importance of such sounds. Third heart sounds should thus be clearly documented as such, particularly when their supposed presence is used to diagnose "heart failure" or made an indication for drug administration." Finally, it is increasingly being recognised that disturbed diastolic function is clinically important in patients with heart disease; our observations show that interrelations between heart sounds and blood flow may provide useful information and may extend simple clinical approaches to this important area of cardiology.

1 Vancheri F, Gibson D. Relation of third and fourth heart sounds to blood velocity during left ventricular filling. $\mathrm{Br}$ Heart J 1989;61:144-8.

2 Pozzoli M, Febo O, Tramarin R, Pinna G, Cobelli F, Specchia G. Pulsed Doppler evaluation of left ventricular filling in subjects with pathologic and physiologic third heart sound. Eur Heart J 1990;11:500-8.

3 Sasson Z, Hatle L, Appleton CP, Jewitt M, Alderman EL, Popp RL. Intracardiac flow during isovolumic relaxation: description and characterization by Doppler echocardiography. J Am Coll Cardiol 1987;10:539-46.

4 Gibson DG, Prewitt TA, Brown DJ. Analysis of left ventricular wall movement during isovolumic relaxation
valt ventricular wall movement during isovolumic relaxation and its relation

5 Lee DCS, Johnson RA, Bingham JB, et al. Heart failure in outpatients. A randomised trial of digoxin versus placebo.
$N$ Engl J Med 1982;306:699-705. 